\title{
Control of the innate immune response by the mevalonate pathway
}

\author{
Murali K Akula ${ }^{1-3,11}$, Man Shi ${ }^{1,2,11}$, Zhaozhao Jiang4,11, Celia E Foster4,11, David Miao ${ }^{1,2}$, Annie S Li ${ }^{4}$, \\ Xiaoman Zhang 4 , Ruth M Gavin ${ }^{4}$, Sorcha D Forde ${ }^{4}$, Gail Germain ${ }^{4}$, Susan Carpenter ${ }^{4}$, Charles V Rosadini ${ }^{5}$, \\ Kira Gritsman ${ }^{6,7}$, Jae Jin Chae ${ }^{8}$, Randolph Hampton ${ }^{9}$, Neal Silverman ${ }^{4}$, Ellen M Gravallese ${ }^{10}$, Jonathan C Kagan ${ }^{5}$, \\ Katherine A Fitzgerald ${ }^{4}$, Daniel L Kastner ${ }^{8}$, Douglas T Golenbock ${ }^{4}$, Martin O Bergo ${ }^{3} \&$ Donghai Wang ${ }^{1,2,4}$
}

\begin{abstract}
Deficiency in mevalonate kinase (MVK) causes systemic inflammation. However, the molecular mechanisms linking the mevalonate pathway to inflammation remain obscure. Geranylgeranyl pyrophosphate, a non-sterol intermediate of the mevalonate pathway, is the substrate for protein geranylgeranylation, a protein post-translational modification that is catalyzed by protein geranylgeranyl transferase I (GGTase I). Pyrin is an innate immune sensor that forms an active inflammasome in response to bacterial toxins. Mutations in MEFV (encoding human PYRIN) result in autoinflammatory familial Mediterranean fever syndrome. We found that protein geranylgeranylation enabled Toll-like receptor (TLR)-induced activation of phosphatidylinositol-3-OH kinase (PI(3)K) by promoting the interaction between the small GTPase Kras and the PI(3)K catalytic subunit p1108. Macrophages that were deficient in GGTase I or p110反 exhibited constitutive release of interleukin $1 \beta$ that was dependent on MEFV but independent of the NLRP3, AIM2 and NLRC4 inflammasomes. In the absence of protein geranylgeranylation, compromised $\mathrm{PI}(3) \mathrm{K}$ activity allows an unchecked TLR-induced inflammatory responses and constitutive activation of the Pyrin inflammasome.
\end{abstract}

The mevalonate pathway is a fundamental metabolic pathway responsible for cholesterol biosynthesis that has been successfully targeted by statins, which are inhibitors of the 3-hydroxyl-3-methylglutaryl co-enzyme A reductase (HMGCR), in the treatment of hypercholesterolemia. MVK catalyzes the phosphorylation of mevalonate, a crucial step in the mevalonate pathway ${ }^{1}$. Patients carrying loss-of-function mutations in $M V K$ develop inflammatory phenotypes, including recurrent fever, lymphadenopathy, arthritis and elevated inflammatory cytokines in serum. There are two types of MVK deficiency, the less-severe hyperimmunoglobuline $\mathrm{D}$ and periodic fever syndrome (HIDS) and the more severe mevalonate aciduria (MA) ${ }^{2}$. The autoinflammatory phenotypes in patients with mevalonate-kinase deficiency (MKD) suggest that the mevalonate pathway critically regulates the inflammatory response, but the underlying molecular mechanisms remain unknown.

Unlike the conventional inflammatory cytokines, interleukin $1 \beta$ (IL-1 $\beta$ ) and the closely related interleukin-18 (IL-18) are atypical in that they are synthesized as inactive precursors in the cytosol ${ }^{3}$. Maturation and release of these cytokines depends on inflammasomes, large protein complexes that consist of a receptor, the adaptor protein ASC and pro-inflammatory caspases ${ }^{4}$. Dysregulation of inflammasome activity and hyper-production of IL- $1 \beta$ are associated with autoinflammatory diseases ${ }^{5}$. One such disease, familial Mediterranean fever (FMF), is caused by mutations in $M E F V$, which encodes human PYRIN, an innate immune sensor that forms a caspase-1-activating inflammasome in response to bacterial toxins ${ }^{6}$. These FMF-associated mutant forms of PYRIN confer a heightened sensitivity to PYRIN inflammasome, leading to hyper-production of IL- $1 \beta$ and autoinflammation.

The PI(3)Ks also have important roles in the regulation of the innate immune response ${ }^{7,8}$. Kinases downstream of PI(3)Ks, such as Akt1 (ref. 9), glycogen synthase kinase- $3 \beta(\text { Gsk } 3 \beta)^{10}$ and the mechanistic Target of Rapamycin (mTOR) ${ }^{11}$, inhibit TLR-induced pro-inflammatory cytokine production through post-translational modification of transcription factors such as NF- $\kappa \mathrm{B}, \mathrm{AP}-1, \mathrm{CREB}$ and STAT3 (refs. 12,13). $\mathrm{PI}(3) \mathrm{K}$ activity is regulated by multiple factors, including Ras and Rho family small GTPases ${ }^{14}$.

In addition to cholesterols, the mevalonate pathway also synthesizes other intermediates, such as the non-sterol isoprenoid geranylgeranyl pyrophosphate (GGPP). GGPP can be used as substrate for

\footnotetext{
${ }^{1}$ Division of Rheumatology and Immunology, Department of Medicine, Duke University School of Medicine, Durham, North Carolina, USA. ${ }^{2}$ Department of Immunology, Duke University School of Medicine, Durham, North Carolina, USA. ${ }^{3}$ Sahlgrenska Cancer Center, Department of Molecular and Clinical Medicine, Institute of Medicine, University of Gothenburg, Gothenburg, Sweden. ${ }^{4}$ Division of Infectious Diseases and Immunology, the University of Massachusetts Medical School, Worcester, Massachusetts, USA. ${ }^{5}$ Division of Gastroenterology, Boston Children's Hospital and Harvard Medical School, Boston, Massachusetts, USA. ${ }^{6}$ Department of Medicine, Albert Einstein College of Medicine, New York City, New York, USA. ${ }^{7}$ Department of Cell Biology, Albert Einstein College of Medicine, New York City, New York, USA. 8 Inflammatory Disease Section, Metabolic, Cardiovascular and Inflammatory Disease Genomics Branch, National Human Genome Research Institute, National Institutes of Health, Bethesda, Maryland, USA. ${ }^{9}$ Division of Biology, University of California San Diego, La Jolla, California, USA. ${ }_{10}$ Division of Rheumatology, Department of Medicine, the University of Massachusetts Medical School, Worcester, Massachusetts, USA. 11These authors contributed equally to this work. Correspondence should be addressed to D.W. (donghai.wang@duke.edu).
} 
protein post-translational modifications; namely, protein geranylgeranylation $^{15}$. Protein geranylgeranylation is catalyzed by GGTase I. Macrophages that are deficient in the $\beta$-subunit of GGTase I (protein geranylgeranyl transferase-1 $\beta$ subunit; encoded by Pggt1b) are hyper-activated by lipopolysaccharide (LPS). Mice with conditional deficiency in Pggt1b in myeloid cells also develop inflammatory arthritis spontaneously ${ }^{16}$. These observations suggest that protein geranylgeranylation controls the innate immune response; however, the underlying molecular mechanisms remain unclear.

We uncovered an unanticipated link between the mevalonate pathway and TLR-induced PI(3)K activation. Through protein geranylgeranylation, the mevalonate pathway enabled TLR-induced PI(3)K activation by promoting the interaction between the Ras family small GTPase Kras and the PI(3)K catalytic subunit p110 $\delta$. Inhibition of the mevalonate pathway decreased the production of GGPP and limited protein geranylgeranylation, thereby impairing $\mathrm{PI}(3) \mathrm{K}$ activation. Impaired PI(3)K activation resulted in hyper-inflammatory conditions, including hyper-production of pro-inflammatory cytokines, elevated expression of Pyrin protein and spontaneous activation of the Pyrin inflammasome.

\section{RESULTS}

\section{Differential regulation of cytokine production by Pggt1b}

To define the role of protein geranylgeranylation in the regulation of TLR-induced production of cytokines, we used enzyme-linked immunosorbent assay (ELISA) to assess the response of Pggt1b-deficient bone-marrow-derived macrophages (BMDMs) to stimulation by ligands of TLRs. Stimulation of Pggt1b-deficient macrophages with ligands to TLR4 (LPS), TLR9 (CpG), TLR2 (Pam3CSK4) and TLR7 (R848) led to potent upregulation of pro-inflammatory cytokines such as IL-1 $\beta$, TNF, IL-6 and IL-12 in comparison with wild-type control BMDMs (Fig. 1a-d). Concurrently, the production of anti-inflammatory IL-10, as well as type I interferon and the chemokine RANTES, was suppressed in Pggt1b-deficient macrophages (Fig. 1e-g). Notably, Pggt1b-deficient BMDMs exhibited decreased production of both pro- and anti-inflammatory cytokines following stimulation with the TLR3 ligand poly(I:C). Quantitative RT-PCR revealed that the altered cytokine production in Pggtlb-deficient cells was caused by altered levels of transcription (Supplementary Fig. 1a), suggesting that protein geranylgeranylation differentially regulates cytokine gene expression at the transcriptional level. In addition to the commonly studied cytokines, we also observed enhanced production of granulocyte growth factor G-CSF and neutrophil chemotactic factor
$\mathrm{KC}(\mathrm{Cxcl} 1)$, as well as dampened production of chemokines MCP-1, MIP $1 \beta$ and MIP $1 \alpha$, in a multiplex-cytokine assay (Supplementary Fig. 1b). Pggt $1 b^{\mathrm{fl} / \mathrm{fl}} L y z 2$-Cre mice had elevated frequencies of granulocytes in their circulation (Fig. 2a,b). To further investigate the physiological relevance of altered cytokine production caused by Pggtlb deficiency in vivo, we challenged mice with LPS at a lethal dose of $30 \mathrm{mg}$ per $\mathrm{kg}$ of body weight. Within $12 \mathrm{~h}$ of LPS challenge, $80 \%$ of Pggt $1 b^{\mathrm{fl} / \mathrm{fl}}$ Lyz2-Cre mice died, whereas none of the Pggt $1 b^{\mathrm{fl} /+}$ Lyz2-Cre mice died (Fig. 2c). The increased susceptibility of mice to endotoxic shock correlated with higher LPS-induced serum concentrations of the pro-inflammatory cytokines IL-1 $\beta$, TNF and IL-6 (Fig. 2d-f). Together these data suggest that Pggt1b differentially regulate TLRinduced inflammatory cytokine production.

IKK and MAPK activation are not affected by Pggt1b deficiency The altered cytokine production in Pggt1b-deficient macrophages prompted us to evaluate the activation of the IKK, MAPK and TBK1 signaling pathways downstream of TLRs ${ }^{17}$. We stimulated wild-type and Pggt $1 \mathrm{~b}$-deficient macrophages with a low dose of LPS $(10 \mathrm{ng} / \mathrm{ml})$ and evaluated the activation of IKK $\beta$, MAPK and TBK1 by immunoblot analysis with phosphorylation-specific antibodies. Activation of IKK $\beta$, JNK, p38, Erk and TBK1 was not enhanced or suppressed in Pggt1b-deficient macrophages compared with their activation in wildtype control cells (Supplementary Fig. 2a,b). Endocytosis of TLR4 induced by LPS stimulation was not impaired or enhanced in Pggt1bdeficient macrophages either (Supplementary Fig. 2c), suggesting that there is no TLR trafficking defect ${ }^{18}$. We conclude that the altered cytokine profile in Pggtlb-deficient macrophages is not a result of altered activation of IKK, MAPK, TBK1 or TLR endocytosis.

\section{Compromised $\mathrm{PI}(3) \mathrm{K}$ activation in the absence of Pggt1b}

The cytokine production profile that we observed in Pggt1b-deficient macrophages is markedly similar to that in myeloid cells deficient in $\mathrm{PI}(3) \mathrm{K}$-Akt signaling components, such as $\mathrm{p} 85 \alpha^{19,20}, \mathrm{p} 110 \delta^{18}$, mTOR $^{11}$ and Akt1 (ref. 9), or treated with Gsk3 $\beta$ inhibitors ${ }^{10}$. We speculated that protein geranylgeranylation regulates TLR-induced cytokine production by controlling PI(3)K-Akt activation. Pggt1bdeficient macrophages displayed substantially lower constitutive Akt phosphorylation. When Pggt1b-deficient BMDMs were stimulated with LPS, phosphorylation of Akt at both Ser473 and Thr308 was severely compromised compared with wild-type control cells (Fig. 3a). Consistent with compromised Akt activation, phosphorylation of Gsk3 $\beta$ at Ser9 was also severely diminished in Pggt1b-deficient cells
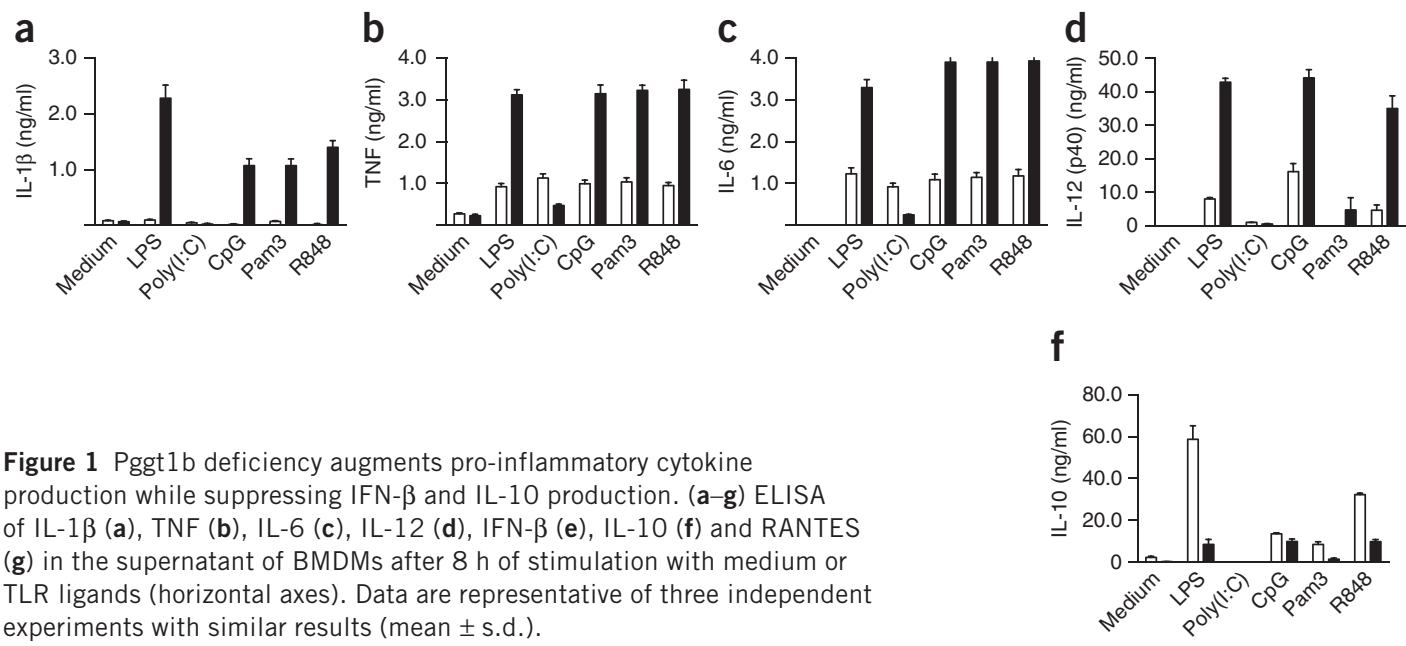

Figure 1 Pggt 1 b deficiency augments pro-inflammatory cytokine production while suppressing IFN- $\beta$ and IL-10 production. (a-g) ELISA of IL-1 $\beta$ (a), TNF (b), IL-6 (c), IL-12 (d), IFN- $\beta$ (e), IL-10 (f) and RANTES (g) in the supernatant of BMDMs after $8 \mathrm{~h}$ of stimulation with medium or TLR ligands (horizontal axes). Data are representative of three independent experiments with similar results (mean \pm s.d.).

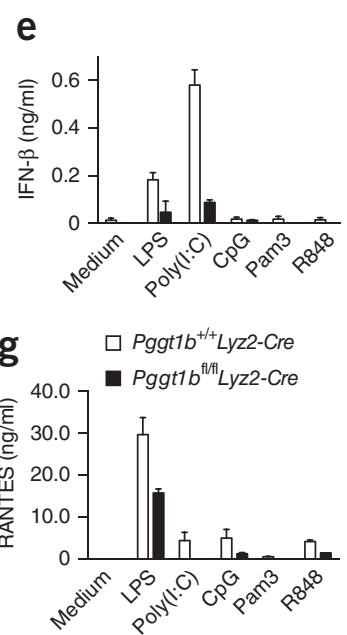


a

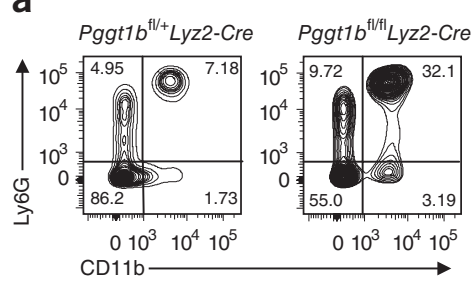
Pggt1 $b^{\mathrm{ft} / 1 / 1}$ Lyz2-Cre

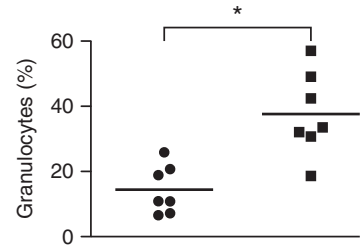

C

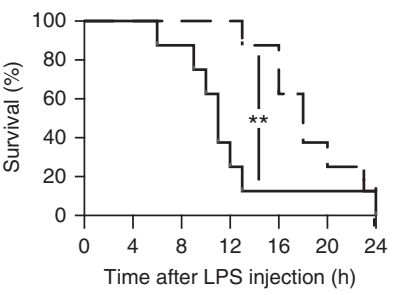

d $\quad$ Pggt1 ${ }^{\mathrm{fl} / \mathrm{L}}$ Lyz2-Cre

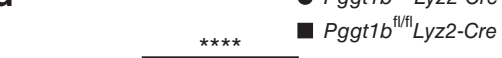

Figure 2 Neutrophilia and enhanced susceptibility to endotoxic shock in Pggt $1 b^{f / / f l} L y z 2-C r e$ mice. (a) Flow cytometry of CD11 b+ Ly6G+ granulocytes in the peripheral blood of Pggt $1 b^{f \mid /+}$ Lyz2-Cre and Pggt $1 b^{\mathrm{fl} / f \mid} L y z 2$-Cre mice. (b) Frequency of granulocytes in sex- and age-matched control Pggt $1 b^{\mathrm{fl} /+}$ Lyz2-Cre $(n=7)$ and Pggt1 $b^{\mathrm{fl} / f \mid} L y z 2$-Cre $(n=7)$ mice. (c) Survival of Pggt $1 b^{\mathrm{f} / /+} L y z 2-C r e$ (dotted line) $(n=8)$ and Pggt $1 b^{f|f|} L y z 2-C r e$ (solid line) $(n=8)$ mice injected with a lethal dose of LPS. (d-f) ELISA of IL-1 $\beta$ (d), TNF (e) and IL-6 (f) in serum from Pggt $1 b^{\mathrm{fl} /+}$ Lyz2-Cre and Pggt $1 b^{\mathrm{f} / f \mid}$ Lyz2-Cre mice $2 \mathrm{~h}$ after LPS injection. Each symbol $(\mathbf{b}, \mathbf{d}-\mathbf{f})$ represents an individual mouse; small horizontal lines indicate the mean. ${ }^{*} P=0.0 .011,{ }^{* *} P=0.0061,{ }^{* * *} P=0.0006$ and ${ }^{* * * *} P<0.0001$ (two-way analysis of variance (ANOVA)). Data are representative of two $(\mathbf{a}, \mathbf{b})$ or three $(\mathbf{c}-\mathbf{f})$ independent experiments.

(Fig. 3b). Gsk3 $\beta$ is phospho-inactivated by Akt at Ser9 (ref. 21). Thus, decreased Ser9 phosphorylation correlates with higher Gsk3 $\beta$ kinase activity. Indeed, immunoblot analysis revealed that phosphorylation of the c-Jun Thr239 and RelA Ser536, two well-established Gsk3 $\beta$ substrates $^{21}$ (Supplementary Fig. 3a), was enhanced in Pggt1bdeficient BMDMs compared with wild-type control cells (Fig. 3b). Phosphorylation of Thr389 on p70S6K kinase, a direct substrate of mTORC1, was greatly decreased in Pggt1b-deficient cells (Fig. 3c). Accordingly, pre-treatment of Pggt1b-deficient macrophages with a Gsk3 $\beta$ inhibitor (SB216763) partially reversed the phenotypes (Supplementary Fig. 3b-f). On the basis of these results, we conclude

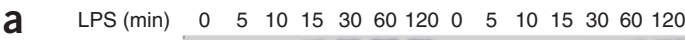
pAkt Ser473 eseeveres - - - - - pAkt Thr308 $\quad-\infty-$

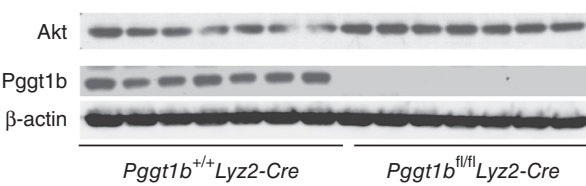

b L LPS (min) $\quad 0 \quad \begin{array}{lllllllllllll}5 & 10 & 15 & 30 & 60 & 120 & 0 & 5 & 10 & 15 & 30 & 60 & 120\end{array}$

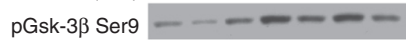

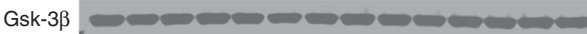
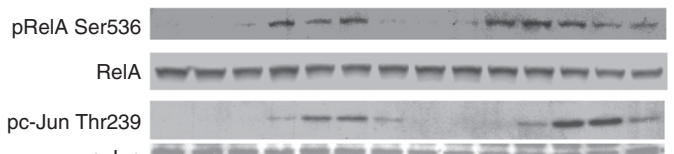

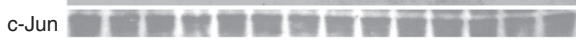

Pggt1b $=-\infty-\infty=$

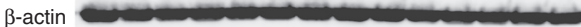

$$
\text { Pggt1 }^{+/+} \text {Lyz2-Cre Pggt1b } b^{\mathrm{fl} / f 1} \text { Lyz2-Cre }
$$

C LPS (min) $\quad 0 \quad \begin{array}{llllllllllllll}5 & 10 & 15 & 30 & 60 & 120 & 0 & 5 & 10 & 15 & 30 & 60 & 120\end{array}$ pp70S6K $\cdots--\cdots=00$ Thr38

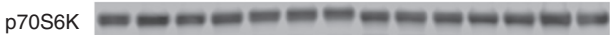

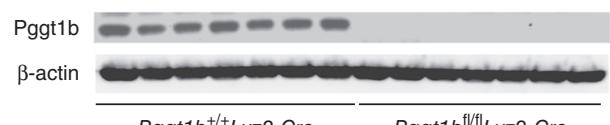

Pggt1 $^{+/+}$Lyz2-Cre Pggt1 $b^{\mathrm{fl} / \mathrm{fl} L y z 2-C r e}$ that Pggt $1 \mathrm{~b}$ controls the PI(3)K-Akt-Gsk $3 \beta$ and mTOR kinase cascade downstream of TLR in macrophages.

\section{Pggt $1 \mathrm{~b}$ deficiency is a phenocopy of $P i k 3 c d^{-/}$BMDMs}

Class IA PI(3)Ks are heterodimers composed of a regulatory subunit


Although $\mathrm{p} 110 \alpha$ and $\mathrm{p} 110 \beta$ are ubiquitously expressed, $\mathrm{p} 110 \delta$ expression is enriched in hematopoietic cells and appears to be more important in immune cell signaling 22 . It has been reported that p110 $\delta$ is involved in balancing the production of pro- versus antiinflammatory cytokines and protects mice from endotoxic shock ${ }^{18}$. We found that LPS stimulation alone induced robust IL- $1 \beta$ release in p1108-deficient ( $P i k 3 c d^{-l-}$ ) BMDMs (Fig. 4a). Pik3cd ${ }^{-/-}$BMDMs also displayed enhanced production of IL-12, TNF and IL- 6 and suppressed production of IFN- $\beta$ (Fig. $4 \mathbf{b}-\mathbf{e}$ ). These phenotypes exactly mirrored those observed in Pggtlb-deficient BMDMs (Fig. 1a). Together, these results suggest that protein geranylgeranylation regulates TLR signaling, most probably through p $110 \delta$ of the class IA PI(3)K family.

\section{Constitutively active $\mathrm{p} 110 \delta$ 'rescues' Pggt1b deficiency}

To further confirm that protein geranylgeranylation controls cytokine production by regulating p $110 \delta$ activation, we infected wild-type or Pggtlb-deficient BMDMs with retrovirus harboring cDNAs encoding wild-type $\mathrm{p} 110 \delta$, Pggt $1 \mathrm{~b}$ or a constitutively active form of $\mathrm{p} 110 \delta$ $(\mathrm{E} 1121 \mathrm{~K})^{23}$. Expression of E $1121 \mathrm{~K}$, but not wild-type p $110 \delta$, completely abrogated LPS-induced IL- $1 \beta$ release and restored the production of

Figure 3 Compromised LPS-induced phosphorylation of Akt, GSK3 $\beta$ and mTOR in the absence of Pggt $1 \mathrm{~b}$. (a) Immunoblot analysis of Akt phosphorylated at Ser473 (pAkt Ser473) or Thr308 (pAkt Thr308) and total Akt and Pggt $1 \mathrm{~b}$, as well as $\beta$-actin (loading control throughout), in lysates of BMDMs stimulated for various times (above lanes) with LPS (10 ng/ml). (b) Immunoblot analysis of Gsk3 $\beta$ phosphorylated at Ser9 (pGsk3 $\beta$ Ser9) and total Gsk3 $\beta$, RelA phosphorylated at Ser536 (pRelA Ser536) and total RelA, and c-Jun phosphorylated at Thr239 (pc-Jun Thr239) and total c-Jun, and Pggt 1b, in lysates of BMDMs stimulated as in a. (c) Immunoblot analysis of p70S6K phosphorylated at Thr389 (p70S6K Thr389) and total p70S6K and Pggt1b in lysates of BMDMs stimulated as in a. Data are from one experiment (same for all panels) representative of three independent experiments with similar results. 
a

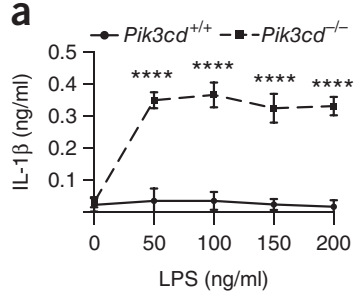

f

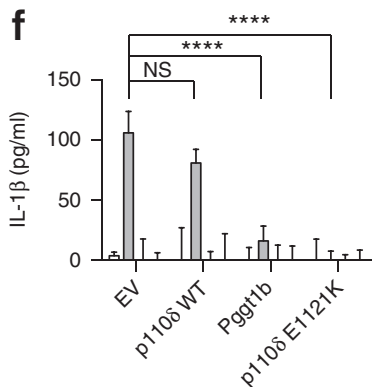

b
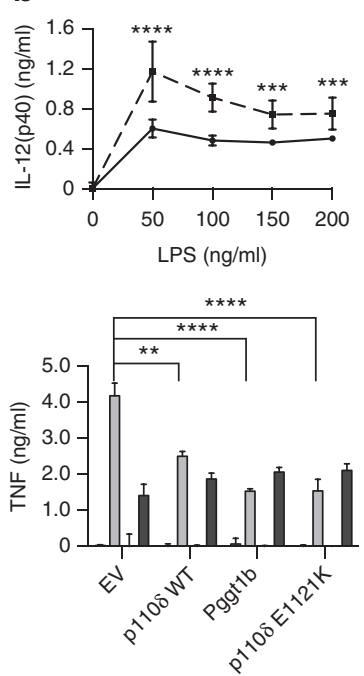

C
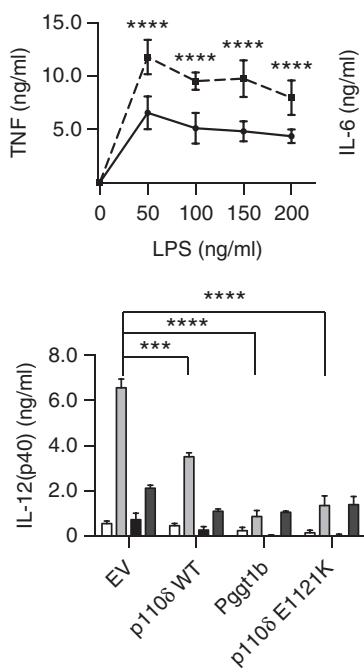

d

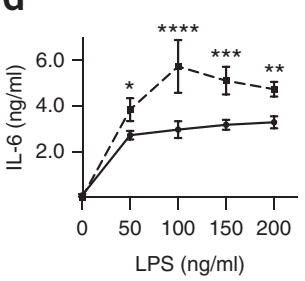

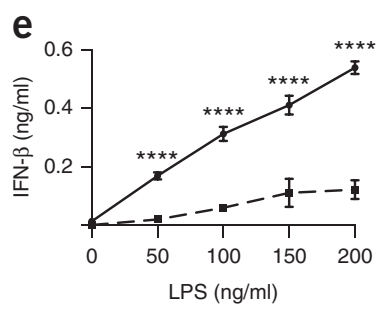

e

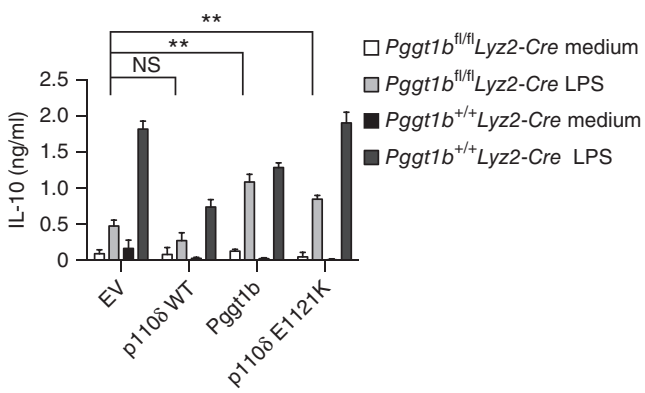

Figure 4 Protein geranylgeranylation controls TLR-induced cytokine production and inflammasome activation through the PI(3)K-p110 . (a-e) ELISA

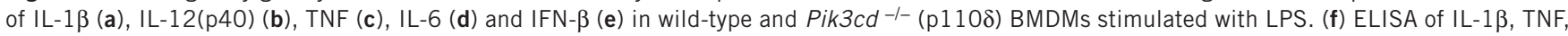
IL-12(p40) or IL-10 in supernatants of wild-type (WT) or Pggt1b-deficient BMDMs reconstituted with empty vector (EV) or harboring cDNAs encoding wild-type $\mathrm{p} 110 \delta$, wild-type Pggt $1 \mathrm{~b}$ or a constitutively active form of $\mathrm{p} 110 \delta$ (E1211K). NS, not significant $(P>0.05) ;{ }^{*} P<0.05,{ }^{*} P<0.01,{ }^{*} * * P<$ 0.001 and ${ }^{* * * *} P<0.0001$ (two-way ANOVA). Data are representative of three independent experiments with similar results (mean \pm s.d.).

TNF, IL-12 and IL-10 to the levels observed in wild-type BMDMs; we found the same result in Pggt1b-deficient BMDMs reconstituted with wild-type Pggt1b (Fig. 4f). These findings further confirm that Pggt 1 b controls $110 \delta$ activity downstream of TLR.

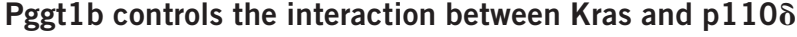

To investigate how protein geranylgeranylation regulates PI(3)K activity, we focused on proteins that can be geranylgeranylated. None of the PI(3)K family members carry a CAAX motif, which is essential for prenylation. However, the catalytic subunits of the class IA PI(3)Ks have a Ras-binding domain (RBD). Ras proteins directly bind to the RBD of the PI(3)K catalytic subunits and activate class I PI(3) $\mathrm{K}^{24}$. The Ras family small GTPases is composed of three members: Hras, Kras and Nras. Computational analysis of farnesylation versus geranylgeranylation potentials using the Prenylation Prediction Suite ${ }^{25}$ revealed that murine Kras and Nras tended to be geranylgeranylated, whereas Hras was unlikely to be modified by geranylgeranylation (GGTase I: Hras, -7.02; Nras, 1.415; Kras, 2.638; farnesyl transferase (FTase) I: Hras, a



Figure 5 Protein geranylgeranylation controls the Kras-p110 interaction that licenses TLR-induced p110 $\delta$ activation. (a) Autoradiography of in vivo labeling of Ras proteins immunoprecipitated from wild-type or Pggt1b-deficient BMDMs. (b) Real-time RT-PCR analysis of Hras, Kras and Nras transcripts (key) in mouse BMDMs $48 \mathrm{~h}$ after transfection with control siRNA or siRNA directed against Hras, Kras or Nras (horizontal axis); results are presented relative to those of the control gene Gapdh. (c) Immunoblot analysis of Akt phosphorylated at Ser473 and total (pan-) Akt in BMDMs transfected with siRNA as in $\mathbf{b}$ and $48 \mathrm{~h}$ later, stimulated for various times (below lanes) with LPS. (d) Quantitation of Akt phosphorylated at S473 in the LPS-stimulated samples in c, calculated as the ratio of phosphorylated Akt to total Akt and presented relative to the ratio in cells treated with control siRNA (Scramble), set as 100\%. (e) Immunoblot analysis of lysates (Input) or anti-p110 $\delta$ immunoprecipitates (IP) of wild-type immortalized macrophages stably infected with EV or pMSCV constructs containing cDNAs encoding wild-type or the geranylgeranylated form (CAAL) of Hras, Kras, and Nras proteins. (f) Immunoblot analysis of anti-p110 $\alpha$, anti-p110 $\beta$ and anti-p1108 immunoprecipitate or lysates of wild-type or Pggt1b-deficient BMDMs stimulated with LPS. Data are representatives of two (a) or three (b-f) independent experiments with similar results.

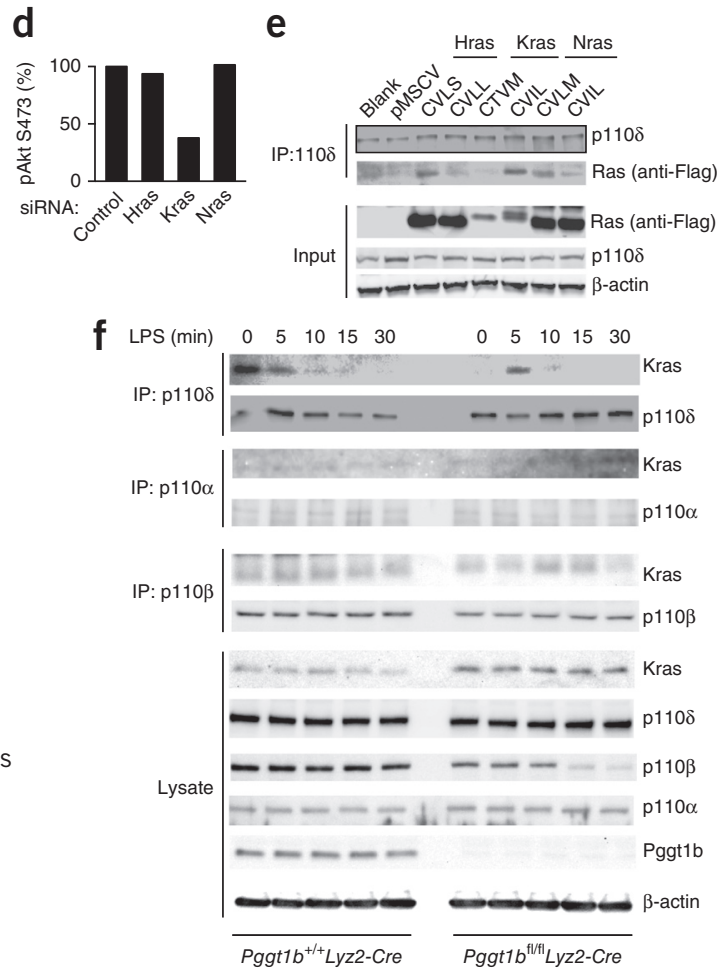


a

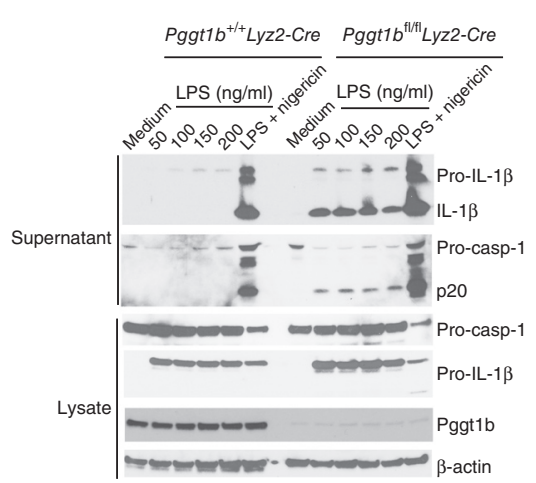

e

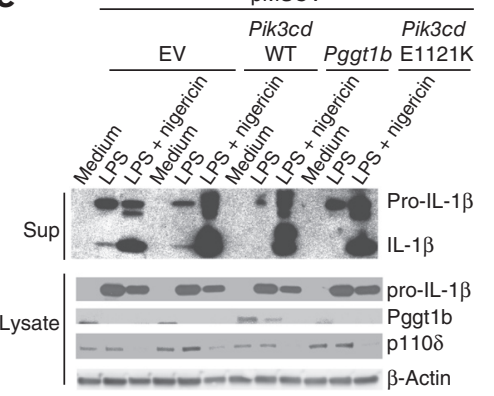

b

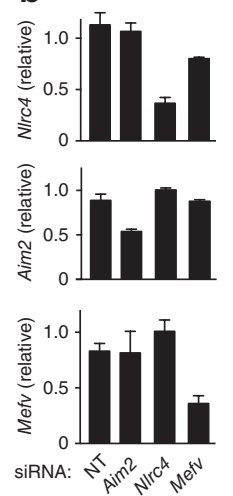

f

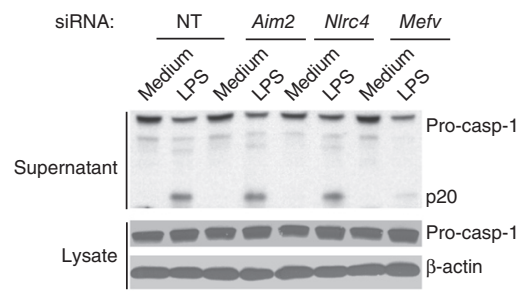

C

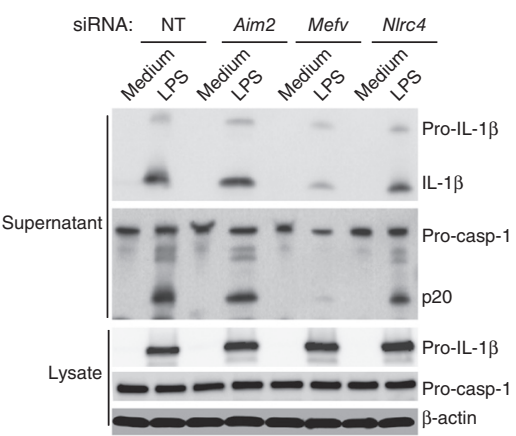

g d

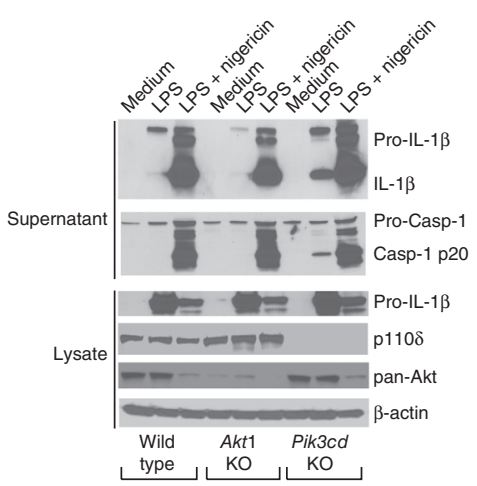

h
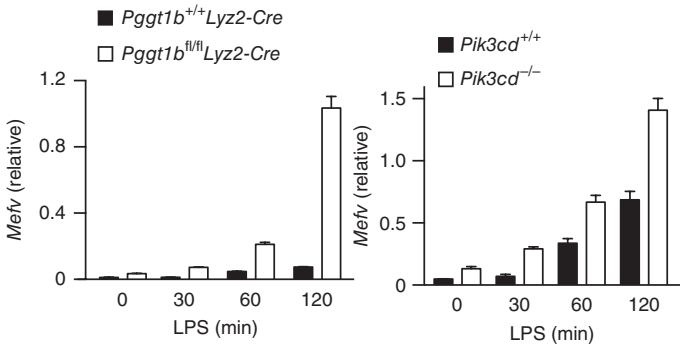

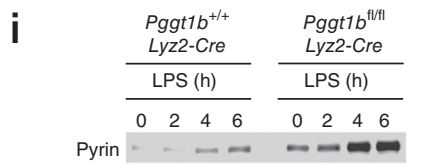

Figure 6 Spontaneous secretion of IL-1 $\beta$ from Pggt $1 b$ and Pik3cd ${ }^{-/-}$macrophages is mediated by the Pyrin
inflammasome. (a) Immunoblot analysis of culture supernatants or lysates of wild-type or Pggt1b-deficient BMDMs stimulated with various concentrations (above lanes) of LPS alone or with LPS plus nigericin.

(b) Real-time RT-PCR analysis of NIrc4, Aim2 and Mefv transcripts in primary BMDMs $48 \mathrm{~h}$ after transfection of control nontargeting siRNA (NT) or siRNA directed against Aim2, NIrc4 or Mefv; results are presented relative to those of the control gene Gapdh. (c) Immunoblot analysis of supernatants or lysates of Pggt 1bdeficient BMDMs 'pre-transfected' with siRNA as in b and stimulated with LPS. (d) Immunoblot analysis of supernatants or lysates of wild-type, $A k t 1^{-1-}$ and Pik3cd-l- BMDMs stimulated with LPS. (e) Immunoblot analysis of supernatants or lysates of Pggt1b-deficient BMDMs reconstituted with EV or harboring cDNAs encoding wild-type $\mathrm{p} 110 \delta$, wild-type Pggt $1 \mathrm{~b}$ or the constitutively active form of $\mathrm{p} 110 \delta$ (E1211K). (f) Immunoblot analysis of Pik3cd ${ }^{-/-}$BMDMs 'pre-transfected' with siRNA as in b stimulated with LPS. (g,h) Real-time RT-PCR analysis of Mefv transcripts in Pggt1b-deficient (g) or Pik3cd -/(h) BMDMs stimulated with LPS; results presented as in b. (i) Immunoblot analysis of Pyrin in lysates of LPS-stimulated Pggt1b-deficient and control BMDMs. Data are representative of three independent experiments with similar results (mean and s.d. in $\mathbf{b}, \mathbf{g}, \mathbf{h}$ ).

1.305; Nras, 0.003; Kras, 1.816). This is consistent with a published report indicating that human KRAS can be geranylgeranylated in the presence of farnesylation inhibitors, whereas HRAS can only be farnesylated ${ }^{26}$. In vivo labeling of primary BMDMs using ${ }^{3} \mathrm{H}$-geranylgeranyl pyrophosphate revealed that murine Ras proteins can be geranylgeranylated in macrophages under physiological conditions and that this process depends on Pggt1b (Fig. 5a).

To evaluate which murine Ras proteins mediate PI(3)K activation, we knocked down the expression of Kras, Nras or Hras in primary BMDMs using small interfering RNA (siRNA). Knocking down the expression of Kras, but not Hras or Nras, in primary BMDMs markedly decreased Akt phosphorylation following LPS stimulation (Fig. 5b-d). We therefore conclude that Kras, but not Hras or Nras, is essential for TLR-induced PI(3)K activation.

In the carboxy-terminal CAAX motifs of prenylated proteins, C represents cysteine, A represents aliphatic amino acid and X can be any amino acid. The carboxyl terminal $(\mathrm{X})$ directs either farnesylation or geranylgeranylation. GGTase I prefers substrates with a carboxyl terminal leucine $(\mathrm{CAAL})^{27}$. To investigate how prenylation regulates Ras GTPases interaction with $\mathrm{p} 110 \delta$, we constructed wild-type and CAAL mutant forms of Ras-encoding cDNAs (wild type: Hras, CVLS; Nras, CVLM; Kras, CTVM; CAAL mutant: Hras, CVLL; Nras, CVIL;
Kras, CVIL) into retroviral vectors. The Prenylation Suite predicted these CAAL mutant Ras GTPases to be more likely to be geranylgeranylated (data not shown). Stable cell lines were established by transduction of immortalized wild-type BMDMs with retroviral vectors harboring cDNAs encoding either wild-type or the CAAL mutant form of Ras proteins. Immunoprecipitation of endogenous $\mathrm{p} 110 \delta$ revealed that interaction of Kras-CVIL (the CAAL (geranylgeranylated) form) with p $110 \delta$ was markedly enhanced in comparison with the wild-type Kras (CTVM) protein. In the same setting of the experiments, the interaction between Nras or Hras with p $110 \delta$ was adversely affected by the increased potential of geranylgeranylation (Fig. 5e). This result suggests that protein geranylgeranylation specifically increases the interaction between Kras and p $110 \delta$.

Given that Ras-PI(3)K interaction is essential for optimal PI(3)K activation ${ }^{14}$, we hypothesized that protein geranylgeranylation regulates the Kras-p1 $10 \delta$ interaction. Immunoprecipitation of $\mathrm{p} 110 \delta$ from cell lysate of LPS-stimulated BMDMs revealed that Kras proteins were constitutively associated with p $110 \delta$ in resting wild-type cells. This was consistent with the higher basal level of Akt phosphorylation in wild-type cells (Fig. 3a). Following LPS stimulation, Ras proteins dissociated from p110 (Fig. 5f). However, in Pggt1b-deficient BMDMs, this constitutive association was markedly decreased. 
Figure 7 Enhanced cytokine production and inflammasome activation in PBMCs obtained from patients with HIDS and treated with LPS and simvastatin. (a) Immunoblot analysis of uncleaved (pro-) and cleaved IL-1 $\beta$ in supernatants of PBMCs obtained from healthy control subjects or patients with HIDS (1-6) and stimulated with LPS alone or LPS plus simvastatin. (b) Expression of genes encoding pro-inflammatory cytokines (left margin) in PBMCs obtained from healthy control subjects and stimulated with LPS alone or LPS plus simvastatin. (c) Immunoblot analysis of Akt phosphorylated at Ser473 and total Akt in lysates of human PBMCs pre-treated overnight with the vehicle DMSO or simvastatin $(5 \mu \mathrm{M})$ and then stimulated with LPS. Data are representative of two experiments with similar results.

Although LPS stimulation could induce transient interaction between Kras proteins and $\mathrm{p} 110 \delta$, this interaction was much weaker and was not sustained for more than $10 \mathrm{~min}$ (Fig. $\mathbf{5 f}$ ). We did not see altered interaction between Kras and the class IA catalytic subunits p110 $\alpha$ and $\mathrm{p} 110 \beta$. These observations suggest that the interaction between Kras and $\mathrm{p} 110 \delta$ is regulated by protein geranylgeranylation.

Pyrin inflammasome mediates IL-1 $\beta$ release in Pggt1 $b^{-/-}$BMDMs The success of treating patients with MKD with anti-interleukin-1 agents such as the recombinant IL-1 receptor antagonist anakinra ${ }^{28}$ suggests that IL-1 and inflammasomes are important in the pathogenesis of MKD. Stimulation of Pggt1b-deficient macrophages with LPS alone resulted in robust IL-1 $\beta$ maturation and release, whereas the same stimulation did not induce robust IL-1 $\beta$ production in wildtype cells (Figs. 1a and 6a). The LPS-induced processing and release of IL-1 $\beta$ in Pggt1b-deficient BMDMs began $2 \mathrm{~h}$ after LPS stimulation and peaked at $6 \mathrm{~h}$ (Supplementary Fig. 4a). In addition to LPS, CpG (TLR9), Pam3CSK4 (TLR2) and R848 (TLR7), but not poly(I:C), induced spontaneous IL-1 $\beta$ maturation and release in Pggtlbdeficient BMDMs (Supplementary Fig. 4b), suggesting that TLR3 signaling is differentially regulated by protein geranylgeranylation than other TLRs. Re-expression of Pggt1b in Pggt1b-deficient BMDMs completely reversed the spontaneous IL-1 $\beta$ maturation following LPS stimulation (Supplementary Fig. 4c,d).

Further analysis using BMDMs from compound transgenic strains of mice revealed that IL- $1 \beta$ maturation and release was abrogated in Pggt $1 b^{-/-}$Casp $^{-/-}$or Pggt1 $b^{-/-}$Pycard $^{-1-}$ (Pycard encodes ASC) BMDMs stimulated with LPS, but not in Pggt $1 b^{-1-} \mathrm{Nlrp}^{-/-}$cells (Supplementary Fig. 5a-c). These results indicate that TLR-induced spontaneous IL- $1 \beta$ maturation in Pggt1b-deficient BMDMs is mediated by caspase-1 and ASC, but not by NLRP3. Given that caspase-11mediated IL-1 $\beta$ processing also depends on NLRP3 (ref. 29), we ruled out the possibility of a role for caspase- 11 .

Pyrin has been defined as an innate immune sensor that forms a caspase-1-activating inflammasome in response to bacteria toxins ${ }^{6}$. Mutations in the human PYRIN-encoding MEFV gene result in $\mathrm{FMF}^{5}$. Using an siRNA approach, we found that knocking down the expression of Mefv, but not Aim 2 or Nlrc4, resulted in a marked inhibition of IL-1 $\beta$ release and caspase- 1 processing in Pggt1b-deficient BMDMs (Fig. 6b,c), suggesting that the Pyrin inflammasome mediates LPS-induced IL-1 $\beta$ secretion in Pggt1b-deficient BMDMs. Similar to Pggtlb-deficient cells, stimulation of $P i k 3 \mathrm{~cd}^{-/-}$BMDMs with LPS alone also induced robust IL-1 $\beta$ production (Figs. $4 \mathbf{a}$ and $\mathbf{6 d}$ ). The maturation of IL- $1 \beta$ in Pik3cd ${ }^{-1-}$ BMDMs was accompanied by the release of the caspase- 1 p20 subunit, an indication of the activation of capase-1 inflammasomes (Fig. 6d). Notably, re-expression of a constitutively active form of $\mathrm{p} 110 \delta(\mathrm{E} 1121 \mathrm{~K})$ completely rescued the IL-1 $\beta$-producing phenotype in Pggtlb-deficient BMDMs (Figs. 4f and $6 \mathbf{e}$ ). Furthermore, an siRNA approach revealed that knocking down just the expression of Mefv severely inhibited LPS-induced

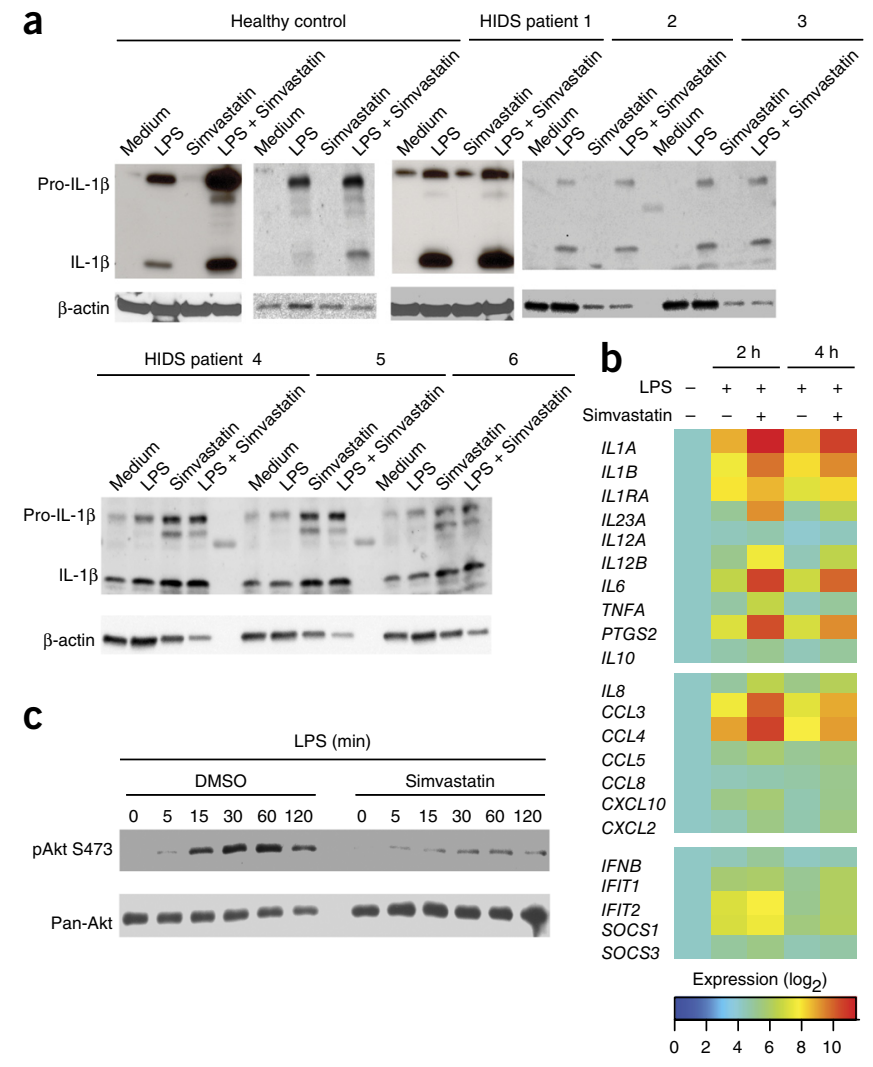

inflammasome activation in $P i k 3 c d^{-1-}$ BMDMs (Fig. 6f). Taken together, these data indicate that GGTase I controls Pyrin inflammasome activation through the $\mathrm{PI}(3) \mathrm{K}-\mathrm{p} 110 \delta$ signaling pathway.

\section{Pggt $1 \mathrm{~b}$ and $\mathrm{p} 110 \delta$ control Mefv expression}

We found that Mefv mRNA expression was barely detectable in wildtype BMDMs without stimulation, and its expression was induced by LPS treatment. In the Pggt1b- or p110 $\delta$-deficient BMDMs, the basal level of Mefv mRNA expression was higher than that of wild-type controls. Notably, Mefv expression was hyper-induced in both Pggt1bdeficient BMDMs and p110 $\delta$-deficient cells (Fig. 6g,h). Consistent with that, Pyrin protein expression was much higher in Pggt1bdeficient BMDMs (Fig. 6i). It is known that expression of Mefv is driven by the NF- $\kappa \mathrm{B}$ subunit $\operatorname{RelA}^{30}$ and that the kinase Gsk3 $\beta$ promotes the transactivation activity of RelA via phosphorylation of RelA ${ }^{10}$. We found rampant Gsk3 $\beta$ activity and enhanced phosphorylation of RelA in Pggt1b-deficient BMDMs (Fig. 3b). Taken together, our results support the notion that enhanced NF- $\mathrm{BB}$ RelA transactivational activity drives hyper-expression of Mefv that may contribute to TLR-induced IL- $1 \beta$ release in Pggt1b- or $p 110 \delta$ deficient BMDMs.

\section{Enhanced production of IL-1 $\beta$ in PBMCs from patients with HIDS}

To mimic the disease conditions of patients with HIDS, we used healthy human peripheral blood mononuclear cells (PBMCs) treated with simvastatin, an inhibitor of HMGCR, to inhibit the mevalonate pathway ${ }^{1}$. When we treated healthy PBMCs with LPS, we detected only minimal amounts of IL- $1 \beta$ maturation in the supernatant. Robust IL-1 $\beta$ production only occurred when PBMCs from healthy donors were pre-treated with simvastatin to inhibit the mevalonate pathway (Fig. 7a). However, when PBMCs from patients with HIDS were stimulated with LPS alone, a fraction of the patients showed 
hyper-production of IL-1 $\beta$ without inhibition of the mevalonate pathway by simvastatin (patients 1, 2 and 3 ); in a few other cases, there was spontaneous production of IL- $1 \beta$ without any stimulation, which may have been caused by pathogen infection (patient 4, 5 and 6) (Fig. 7a). Gene-expression profiling revealed that healthy PBMCs, when treated with LPS and simvastatin, displayed enhanced production of proinflammatory cytokines compared with cells stimulated with LPS alone (Fig. 7b). Accordingly, when healthy PBMCs were treated with simvastatin and LPS, phosphorylation of Akt Ser473 was severely compromised (Fig. 7c). These results corroborate our findings in the mouse model of protein geranylgeranylation deficiency and highlight how the mevalonate pathway controls the innate immune response by controlling $\mathrm{PI}(3) \mathrm{K}$-Akt signaling by protein geranylgeranylation in both mouse and human cells (Supplementary Fig. 6).

\section{DISCUSSION}

The crucial role of the PI(3)K-Akt-Gsk3 $\beta$ and mTOR kinase cascade in the regulation of TLR-induced cytokine production has emerged as an important aspect of innate immune signal transduction ${ }^{8,12,21}$. However, the underlying molecular mechanisms are not completely understood. B-cell adaptor for $\mathrm{PI}(3) \mathrm{K}$ (BCAP) is required for $\mathrm{PI}(3) \mathrm{K}$ activation induced by MyD88-dependent TLR4, TLR7 and TLR9 (refs. 18,31). It is not clear whether endosomal TLR3-induced PI(3)K activation also depends on BCAP. TLR3 activates $\mathrm{PI}(3) \mathrm{K}$ by recruiting $\mathrm{PI}(3) \mathrm{K}$ complex directly to its cytoplasmic tails ${ }^{32}$. We found decreased production of IFN- $\beta$ in Pggt1b-deficient BMDMs stimulated with LPS and poly(I:C). Pggt1b-deficient BMDMs displayed attenuated PI(3)K activation following LPS and poly(I:C) (data not shown) stimulation. This is consistent with published data that $\mathrm{PI}(3) \mathrm{K}$ positively regulates IFN- $\beta$ production through inactivation of Gsk $3 \beta^{33-35}$. It is also of note that, unlike ligands to TLR2, 4, 7 and 9, TLR3 ligand poly (I:C) stimulation induced TNF and IL-6 were decreased in Pggtlbdeficient BMDMs. Poly(I:C) also failed to activate inflammasome in the absence of Pggt1b. These results indicate that protein geranylgeranylation differentially regulates MyD88-dependent and MyD88-independent signaling pathways; the precise underlying mechanism merits further investigation.

Published studies have shown that binding of Ras proteins to class IA PI(3)K RBD is essential for both physiological and pathological functions of $\mathrm{PI}(3) \mathrm{Ks}^{24,36}$. However, the manner in which the interaction between Ras and PI(3)K contributes to the TLR-induced innate immune response is not completely understood. In the early 1990s, the finding that farnesylation of KRAS is essential for its oncogenic function galvanized tremendous enthusiasm in developing FTase inhibitors to treat human cancers with KRAS mutations. Unfortunately, the FTase inhibitors showed poor efficacy in clinical trials $^{37}$, as KRAS can also be geranylgeranylated when FTase I is inhibited $^{38-40}$. FTase I and GGTase I are both hetero-dimmers that share an $\alpha$ subunit and have distinct, but highly homologous, $\beta$ subunits ${ }^{15}$. Our data revealed that murine Ras proteins are geranylgeranylated by GGTase I in wild-type mouse BMDMs. In earlier studies, knockout of the $\beta$ subunit of FTase I (Fntb) was shown to block farnesylation of proteins, but had no effect on Kras signaling, membrane targeting or function, and had no effect on cytokine production in myeloid cells ${ }^{16}$. Moreover, the absence of Fntb in macrophages did not induce any inflammatory phenotypes in vivo. Mice deficient in both Pggt $1 b$ and Fntb in macrophages display phenotypes that are indistinguishable from that of the Pggt 16 single-knockout mouse ${ }^{16}$. Taken together, our data suggested that protein geranylgeranylation, but not farnesylation, positively regulates a Kras and p $110 \delta$ interaction that is essential for the activation of $\mathrm{p} 110 \delta$ and its downstream kinase cascades.
It has been shown that deficiency in p $110 \delta$ in dendritic cells causes a defect in intracellular trafficking of TLR4 following LPS stimulation ${ }^{18}$. However, we found no such defect in Pggt1b-deficient BMDMs. Such discrepancies can be attributed to either differences in the cell types examined (macrophage versus DCs) or in the genetic backgrounds of the mouse strains used. Although we saw enhanced production of pro-inflammatory cytokines in healthy PBMCs treated with LPS and simvastatin, the production of INF- $\beta$ and IL-10 was not consistent with that observed in Pggt1b-deficient mouse BMDMs. We believe the discrepancy is caused by the difference of the experimental settings. The phenotype observed in Pggt1b-deficient mouse BMDMs was caused solely by the deficiency in protein geranylgeranylation, whereas simvastatin inhibition of the mevalonate pathway results in the shortage of not only GGPP, but also a variety of other intermediates downstream of HMGCR in the metabolic pathway. The effect of the shortage of those intermediates on inflammatory responses remains to be investigated, but could explain why simvastatin inhibition does not completely recapitulate the phenotypes caused by protein geranylgeranylation deficiency.

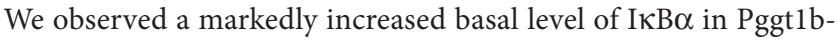
deficient cells. I $\kappa \mathrm{B} \alpha$ itself is a target of NF- $\kappa \mathrm{B}$. Rampant Gsk $3 \beta$ kinase activity caused an increased level of NF- $\mathrm{\kappa} /$ RelA phosphorylation as a result of Pggt1b deficiency leading to an inhibited constitutive level of Akt activity. This in turn increased the transactivational activity of RelA to drive the expression of $I \kappa B \alpha$ gene in resting Pggtlb-deficient cells.

The PI(3)K-Akt-Gsk3 $\beta$ and mTOR pathway have pivotal roles in controlling a variety of essential cellular functions such as cell growth, survival, differentiation and metabolism ${ }^{41}$. Dysregulation of PI(3)K activity is implicated in a myriad of human diseases, particularly cancer and diabetes. Our data implicate protein geranylgeranylation in the regulation of TLR-induced p $110 \delta$ activation in myeloid cells. It is possible that the same mechanisms exist in other tissues and cell types to control class IA PI(3)Ks downstream of growth factor receptors. Thus, our findings may provide clues for manipulating the mevalonate pathway for the intervention of diseases such as cancer, diabetes and aging.

\section{METHODS}

Methods and any associated references are available in the online version of the paper.

Note: Any Supplementary Information and Source Data files are available in the online version of the paper.

\section{ACKNOWLEDGMENTS}

We thank S. Abusneineh for technical support, K. Halmen for lab management, and colleagues in the Golenbock, Fitzgerald and Kastner laboratories for helpful discussions. We also thank M. Birnbaum (University of Pennsylvania) for the Akt1-deficient mice femurs and M. Trombly for editing the manuscript. We are in debt to R. Finberg and E. St. Clair for critical reading of the manuscript. This work was supported in part by funds from the US National Institutes of Health (National Institute of Allergy and Infectious Diseases 1R01AI110695-01A1 to D.W.), an Innovative Research Grant from the Arthritis Foundation (D.W. and E.M.G.), the Swedish Research Council, and the Heart and Lung Foundation (M.B. and M.A.).

\section{AUTHOR CONTRIBUTIONS}

D.W. conceived and designed the study, conducted most of the experiments and drafted the manuscript. A.S.L., C.E.F., M.K.A., M.S., Z.J., C.V.R., J.C.K., S.C., R.M.G., X.Z. and S.D.F. conducted experiments and/or provided technical support. D.M. conducted experiments and provided lab management. G.G. provided animal colony maintenance and performed experiments. K.G. provided $P i k 3 \mathrm{~cd}^{-1-}$ mouse femurs and critically read the manuscript. J.J.C. and D.L.K. provided lab space at the US National Institutes of Health and help with human studies. R.H. contributed to helpful discussion. N.S., E.M.G. and K.A.F. reviewed primary data and critically read the manuscript. D.T.G. provided lab space, reviewed primary data 
and contributed to helpful discussions. M.O.B. provided the Pggt1 $b^{\mathrm{fl} / \mathrm{f}} \mathrm{Lyz2}$-Cre mouse strain, reviewed primary data and contributed to the preparation of the manuscript.

\section{COMPETING FINANCIAL INTERESTS}

The authors declare no competing financial interests.

Reprints and permissions information is available online at http://www.nature.com/ reprints/index.html.

1. Goldstein, J.L. \& Brown, M.S. Regulation of the mevalonate pathway. Nature 343, 425-430 (1990).

2. Frenkel, J. et al. Mevalonate kinase deficiency and Dutch type periodic fever. Clin. Exp. Rheumatol. 18, 525-532 (2000).

3. Dinarello, C.A. Interleukin-1 in the pathogenesis and treatment of inflammatory diseases. Blood 117, 3720-3732 (2011).

4. Martinon, F., Mayor, A. \& Tschopp, J. The inflammasomes: guardians of the body. Annu. Rev. Immunol. 27, 229-265 (2009).

5. Kastner, D.L., Aksentijevich, I. \& Goldbach-Mansky, R. Autoinflammatory disease reloaded: a clinical perspective. Cell 140, 784-790 (2010).

6. Xu, H. et al. Innate immune sensing of bacterial modifications of Rho GTPases by the Pyrin inflammasome. Nature 513, 237-241 (2014).

7. Beurel, E., Michalek, S.M. \& Jope, R.S. Innate and adaptive immune responses regulated by glycogen synthase kinase-3 (GSK3). Trends Immunol. 31, 24-31 (2010).

8. Hazeki, K., Nigorikawa, K. \& Hazeki, O. Role of phosphoinositide 3-kinase in innate immunity. Biol. Pharm. Bull. 30, 1617-1623 (2007).

9. Androulidaki, A. et al. The kinase Akt1 controls macrophage response to lipopolysaccharide by regulating microRNAs. Immunity 31, 220-231 (2009).

10. Martin, M., Rehani, K., Jope, R.S. \& Michalek, S.M. Toll-like receptor-mediated cytokine production is differentially regulated by glycogen synthase kinase 3. Nat. Immunol. 6, 777-784 (2005).

11. Weichhart, T. et al. The TSC-mTOR signaling pathway regulates the innate inflammatory response. Immunity 29, 565-577 (2008).

12. Weichhart, T. \& Säemann, M.D. The PI3K/Akt/mTOR pathway in innate immune cells: emerging therapeutic applications. Ann. Rheum. Dis. 67 (suppl. 3), iii70-iii74 (2008).

13. Ohtani, M. et al. Mammalian target of rapamycin and glycogen synthase kinase 3 differentially regulate lipopolysaccharide-induced interleukin-12 production in dendritic cells. Blood 112, 635-643 (2008).

14. Fritsch, R. et al. RAS and RHO families of GTPases directly regulate distinct phosphoinositide 3-kinase isoforms. Cell 153, 1050-1063 (2013).

15. Zhang, F.L. \& Casey, P.J. Protein prenylation: molecular mechanisms and functional consequences. Annu. Rev. Biochem. 65, 241-269 (1996).

16. Khan, O.M. et al. Geranylgeranyltransferase type I (GGTase-I) deficiency hyperactivates macrophages and induces erosive arthritis in mice. J. Clin. Invest. 121, 628-639 (2011).

17. Takeuchi, O. \& Akira, S. Pattern recognition receptors and inflammation. Cell 140, 805-820 (2010)

18. Aksoy, E. et al. The p1108 isoform of the kinase PI(3)K controls the subcellular compartmentalization of TLR4 signaling and protects from endotoxic shock. Nat. Immunol. 13, 1045-1054 (2012).

19. Fukao, T. et al. PI3K-mediated negative feedback regulation of IL-12 production in DCs. Nat. Immunol. 3, 875-881 (2002).

20. Luyendyk, J.P. et al. Genetic analysis of the role of the PI3K-Akt pathway in lipopolysaccharide-induced cytokine and tissue factor gene expression in monocytes/ macrophages. J. Immunol. 180, 4218-4226 (2008).
21. Wang, $H$., Brown, J. \& Martin, M. Glycogen synthase kinase 3: a point of convergence for the host inflammatory response. Cytokine 53, 130-140 (2011).

22. Okkenhaug, K. Signaling by the phosphoinositide 3-kinase family in immune cells Annu. Rev. Immunol. 31, 675-704 (2013).

23. Lucas, C.L. et al. Dominant-activating germline mutations in the gene encoding the $\mathrm{PI}(3) \mathrm{K}$ catalytic subunit $\mathrm{p} 110 \delta$ result in $\mathrm{T}$ cell senescence and human immunodeficiency. Nat. Immunol. 15, 88-97 (2014).

24. Gupta, S. et al. Binding of ras to phosphoinositide 3-kinase p110alpha is required for ras-driven tumorigenesis in mice. Cell 129, 957-968 (2007).

25. Maurer-Stroh, S. et al. Towards complete sets of farnesylated and geranylgeranylated proteins. PLoS Comput. Biol. 3, e66 (2007).

26. Sebti, S.M. \& Hamilton, A.D. Farnesyltransferase and geranylgeranyltransferase inhibitors and cancer therapy: lessons from mechanism and bench-to-bedside translational studies. Oncogene 19, 6584-6593 (2000).

27. Kinsella, B.T., Erdman, R.A. \& Maltese, W.A. Posttranslational modification of Ha-ras p21 by farnesyl versus geranylgeranyl isoprenoids is determined by the $\mathrm{COOH}$-terminal amino acid. Proc. Natl. Acad. Sci. USA 88, 8934-8938 (1991).

28. Galeotti, C. et al. Efficacy of interleukin-1-targeting drugs in mevalonate kinase deficiency. Rheumatology (Oxford) 51, 1855-1859 (2012).

29. Rathinam, V.A. et al. TRIF licenses caspase-11-dependent NLRP3 inflammasome activation by gram-negative bacteria. Cell 150, 606-619 (2012).

30. Papin, S et al. The tumor necrosis factor alpha-dependent activation of the human mediterranean fever (MEFV) promoter is mediated by a synergistic interaction between C/EBP beta and NF kappaB p65. J. Biol. Chem. 278, 48839-48847 (2003).

31. Ni, M. et al. B-cell adaptor for PI3K (BCAP) negatively regulates Toll-like receptor signaling through activation of PI3K. Proc. Natl. Acad. Sci. USA 109, 267-272 (2012).

32. Sarkar, S.N. et al. Novel roles of TLR3 tyrosine phosphorylation and PI3 kinase in double-stranded RNA signaling. Nat. Struct. Mol. Biol. 11, 1060-1067 (2004).

33. Wang, $\mathrm{H}$. et al. IFN-beta production by TLR4-stimulated innate immune cells is negatively regulated by GSK3-beta. J. Immunol. 181, 6797-6802 (2008).

34. Wang, J.T. et al. Glycogen synthase kinase 3 negatively regulates IFN regulatory factor 3 transactivation through phosphorylation at its linker region. Innate Immun. 20, 78-87 (2014).

35. Hrincius, E.R. et al. Phosphatidylinositol-3-kinase (PI3K) is activated by influenza virus vRNA via the pathogen pattern receptor Rig-I to promote efficient type interferon production. Cell. Microbiol. 13, 1907-1919 (2011).

36. Castellano, E. \& Downward, J. Role of RAS in the regulation of PI 3-kinase Curr. Top. Microbiol. Immunol. 346, 143-169 (2010).

37. Berndt, N., Hamilton, A.D. \& Sebti, S.M. Targeting protein prenylation for cancer therapy. Nat. Rev. Cancer 11, 775-791 (2011)

38. James, G.L., Goldstein, J.L. \& Brown, M.S. Polylysine and CVIM sequences of KRasB dictate specificity of prenylation and confer resistance to benzodiazepine peptidomimetic in vitro. J. Biol. Chem. 270, 6221-6226 (1995).

39. Lerner, E.C., Qian, Y., Hamilton, A.D. \& Sebti, S.M. Disruption of oncogenic KRas4B processing and signaling by a potent geranylgeranyltransferase I inhibitor. J. Biol. Chem. 270, 26770-26773 (1995).

40. Rowell, C.A., Kowalczyk, J.J., Lewis, M.D. \& Garcia, A.M. Direct demonstration of geranylgeranylation and farnesylation of Ki-Ras in vivo. J. Biol. Chem. 272 , 14093-14097 (1997)

41. Engelman, J.A., Luo, J. \& Cantley, L.C. The evolution of phosphatidylinositol 3-kinases as regulators of growth and metabolism. Nat. Rev. Genet. 7, 606-619 (2006). 


\section{ONLINE METHODS}

Mice. C57BL/6J mice from The Jackson Laboratory were bred at the University of Massachusetts Medical School. Pggt $1 b^{\mathrm{fl} / \mathrm{fl}}$ Lyz2-Cre mice were generated as described ${ }^{42}$. Pggt $1 b^{\mathrm{fl} / \mathrm{fl} L y z 2-C r e}$ and littermate control Pggt $1 b^{\mathrm{fl} /+} L y z 2-C r e$ or Pggt 1 $b^{+/+}$Lyz2-Cre were generated by first crossing the Pggt $1 b^{\mathrm{fl} / \mathrm{fl}} \mathrm{Lyz2}$-Cre to C57BL6/J, then intercrossing of the resultant Pggt $1 b^{\mathrm{fl} /+} L y z 2$-Cre mice. Littermate controls were used in the experiments. Mouse bones from $\mathrm{Pik} 3 \mathrm{~cd}^{-1-}$ (encoding $\mathrm{p} 110 \delta$ ) mice were provided by K. Gritsman; the mouse strain was originally generated in the laboratory of $\mathrm{J}$. Ihle ${ }^{43}$ and had been backcrossed to C57BL6 background in the Gritsman laboratory. Bones from C57BL6J mice were used as controls for the in vitro experiments depicted in Figures $4 \mathbf{a}-\mathbf{e}$ and 6d and Supplementary Figure 5. Caspase- 1 and Asc-deficient ${ }^{44}$ mice have been described previously. Mouse strains were maintained in specific pathogen-free conditions in the University of Massachusetts Medical School or the animal facility at Duke University, and the animal protocols were carried out in accordance with the guidelines set forth by the Institutional Animal Care and Use Committees of the University of Massachusetts Medical School or Duke University.

Patient blood samples. HIDS patients and healthy controls were included in the study after informed consent under protocols approved by the Institutional Review Board of the University of Massachusetts Medical School, the Duke University and the National Human Genome Research Institute. A total of three healthy control (two male, one female) and six HIDS patients (two males, four females) were used in the experiments depicted in Figure 7. PBMCs were collected by density centrifugation according to the manufacturer's instructions (GE Healthcare).

BMDM culture and stimulation. Bone marrow cells from wild-type and various knockout mice were cultured in DMEM with 10\% FBS and 20\% L929 supernatants. For immunoblot analysis, BMDMs were primed with $200 \mathrm{ng} / \mathrm{ml}$ LPS unless otherwise indicated for $6 \mathrm{~h}$ and then stimulated with nigericin or the vehicle for an additional hour. For controls in Supplementary Figure 6, BMDMs were transfected with poly(dA:dT) $\left(1 \mu \mathrm{g} / 10^{6}\right.$ cells, Sigma-Aldrich) $2 \mathrm{~h}$ after LPS priming and were left for an additional $6 \mathrm{~h}$ before harvesting. For ELISAs, BMDMs were stimulated with TLR ligands for $8 \mathrm{~h}$ before harvested for ELISA. TLR ligands LPS, poly(I:C), Pam3CSK4, R848 and CpG ODN1826 were purchased from InVivogen. In some cases, Gsk3 $\beta$ inhibitors (SB216763, Abcam and XXVII, Millipore) were added $30 \mathrm{~min}$ before TLR stimulation. For reconstitution experiments, BMDM cultures were infected with retrovirus (pMSCV Clontech) harboring cDNAs encoding wild-type or constitutively active form of $\mathrm{p} 110 \delta$ or wild-type Pggt $1 \mathrm{~b}$ on day 4. The BMDM culture was selected in BMDM medium with $2 \mu \mathrm{g} / \mathrm{ml}$ puromycin for 3 consecutive days starting on day 6 . Viable cells were used for subsequent experiments described in Figures 4d and 6e.

siRNA knockdown of gene expression in BMDMs. Day 7 culture of BMDMs were transfected with siGenome smart pool (GE Healthcare Dharmacon) of non-targeting control (D-001206-13-05) or siGeneome Smart Pool against mouse Hras (M-046324-01-0005), Kras (M-043846-02-0005), Nras (M-044216-01-0005), Mefv (M-048693-00-0005), Aim2 (M-044968-01-005),
Nlrc4 (M-055000-01-0005). $48 \mathrm{~h}$ after siRNA transfection, total RNA were extracted and cDNAs were synthesized. Transcript abundance of murine Hras, Kras, Nras, Mefv, Nlrc4 and Aim2 were determined by quantitative PCR. Primers sequences are listed in Supplementary Table 1. The relative abundance of the transcripts was normalized against that of GAPDH. siRNA treated cells were also stimulated with or without LPS for $30 \mathrm{~min}$, cell lysates were prepared and subjected to immunoblot analysis using anti-phosphor-Akt S473 antibody. Quantitation of Akt phosphorylation in Figure 5d was carried out using ImageJ and normalized against pan-Akt bands in the corresponding lane. Phosphorylation of Akt in control, non-targeting siGenome smart pool were used as $100 \%$ and compared with other samples.

ELISA, multiplex assays and quantitative RT-PCR. Cell culture supernatants or sera were analyzed by ELISA for IL-1 $\beta$, TNF, IL-6, IL-12p40, IL-10 (eBiosciences) and Rantes (R\&D systems). A sandwich ELISA for mouse IFN- $\beta$ was performed as described ${ }^{45}$. Cytokines from cell culture supernatants were quantitated using the Bio-Rad Bio-Plex Mouse Cytokine Assay kit. Transcript abundance of cytokines were determined by quantitative PCR using primers listed in Supplementary Table 1, or with the NanoString nCounter Analysis System (Fig. 7b).

Immunoblot analysis. Proteins from the cell culture supernatants were precipitated by methanol-chloroform extraction. Cells were lysed with $1 \%$ NP40 lysis buffer. Antibodies used for immunoblot analysis and immunoprecipitation are listed in Supplementary Table 2. p110 $\delta$ immunoprecipitation was performed using a previously described protocol ${ }^{46}$. The phosphorylation of Akt (Fig. 5d) was quantified through the use of ImageJ software.

Endotoxic shock. Prior to injection, blood samples were taken from mice. A total of 48 mice, 24 male and 24 female, were used in three independent experiments. Mice were then injected intraperitoneal with LPS (SigmaAldrich) at a lethal dose of $30 \mathrm{mg} / \mathrm{kg}$ bodyweight and returned to the cage and closely observed at 1-h intervals. Blood samples were taken again at $2 \mathrm{~h}$ post LPS injection. Survival time of mice was recorded, and survival curves were generated using GraphPad Prism. Plasma was separated from blood samples and inflammatory cytokines were analyzed using ELISA (eBiosciences).

Statistical analysis. Two-way ANOVA multiple-factor comparison was performed using Prism (Graphpad) software.

42. Sjogren, A.K. et al. GGTase-I deficiency reduces tumor formation and improves survival in mice with K-RAS-induced lung cancer. J. Clin. Invest. 117, 1294-1304 (2007).

43. Jou, S.T. et al. Essential, nonredundant role for the phosphoinositide 3-kinase p110delta in signaling by the B-cell receptor complex. Mol. Cell. Biol. 22, 85808591 (2002).

44. Mariathasan, S. et al. Differential activation of the inflammasome by caspase- 1 adaptors ASC and Ipaf. Nature 430, 213-218 (2004).

45. Roberts, Z.J. et al. The chemotherapeutic agent DMXAA potently and specifically activates the TBK1-IRF-3 signaling axis. J. Exp. Med. 204, 1559-1569 (2007).

46. Bonham, K.S. et al. A promiscuous lipid-binding protein diversifies the subcellular sites of toll-like receptor signal transduction. Cell 156, 705-716 (2014). 